\title{
Long-term follow-up of applying autologous bone grafts for reconstructing tympanomastoid defects in functional cholesteatoma surgery
}

\author{
Wei-Che Lan ${ }^{1}$, Ching-Yuan Wang ${ }^{1}$, Ming-Hsui Tsai ${ }^{1}$, Chia-Der Lin ${ }^{\text {Corresp. } 1,2}$ \\ ${ }^{1}$ Department of Otolaryngology Head and Neck Surgery, China Medical University Hospital, Taichung, Taiwan \\ 2 School of Medicine, China Medical University, Taichung, Taiwan \\ Corresponding Author: Chia-Der Lin \\ Email address: d18546@mail.cmuh.org.tw
}

Objectives. This study investigated the long-term surgical outcomes of functional cholesteatoma surgery with canal wall reconstruction using autologous bone grafts as the primary material in patients with acquired cholesteatoma. Subjects and Methods. Medical charts were retrospectively reviewed for all patients admitted to one institution for surgical intervention between 2010 and 2018. We analyzed 66 patients (66 ears) who underwent functional tympanomastoidectomy involving the use of autologous bone grafts for canal wall defect reconstruction. Surgical outcomes were evaluated by comparing preoperative audiometric results with follow-up data (at least 36 months after surgery). Logistic regression analyses were performed to determine prognostic factors related to long-term hearing success. These factors included classification and stage of cholesteatoma, stapes condition, ossicular chain damage, active infection of the middle ear, state of the contralateral ear, preoperative hearing thresholds, gender, and age. Results: The mean follow-up period was 49.2 months. The recidivism rate was $6 \%$ ( 4 of 66 ears). The pure-tone average significantly improved from $50.78 \pm 19.98$ to $40.81 \pm$ $21.22 \mathrm{~dB}$ hearing level ( $\mathrm{HL} ; \mathrm{p}<0.001$ ). Air-bone gaps significantly improved from $26.26 \pm$ 10.53 to $17.58 \pm 8.21 \mathrm{~dB} \mathrm{HL}(p<0.001)$. In multivariate logistic regression analysis, earlystage disease $(p=0.021)$ and pars flaccida cholesteatoma $(p=0.036)$ exhibited statistically significant correlations with successful hearing preservation. Conclusion: Functional cholesteatoma surgery with autologous bone grafts reconstruction is an effective approach to significantly improve hearing with low recidivism rates. Localized disease and pars flaccida cholesteatoma were two independent predictors of successful hearing preservation. 
1 Long-term follow-up of applying autologous bone grafts for reconstructing

2 tympanomastoid defects in functional cholesteatoma surgery

3

4 Wei-Che Lan, MD1, Ching-Yuan Wang, MD¹, Ming-Hsui Tsai, MD¹, Chia-Der Lin, MD,

$5 \mathrm{PhD}^{1,2}$

6 'Department of Otolaryngology Head and Neck Surgery, China Medical University

7 Hospital, Taichung, Taiwan, R.O.C.

8 2School of Medicine, China Medical University, Taichung, Taiwan, R.O.C.

9

10 Corresponding Author:

11 Chia-Der Lin, PhD, Department of Otolaryngology Head and Neck Surgery, China

12 Medical University Hospital, No.2, Yude Rd., North Dist., Taichung City 404, Taiwan

13 (R.O.C.). Email: d18546@mail.cmuh.org.tw

14

15

16

17 


\section{Abstract}

20 Objectives. This study investigated the long-term surgical outcomes of functional

21 cholesteatoma surgery with canal wall reconstruction using autologous bone grafts as

22 the primary material in patients with acquired cholesteatoma.

23 Subjects and Methods. Medical charts were retrospectively reviewed for all patients

24 admitted to one institution for surgical intervention between 2010 and 2018. We

25 analyzed 66 patients (66 ears) who underwent functional tympanomastoidectomy

26 involving the use of autologous bone grafts for canal wall defect reconstruction. Surgical

27 outcomes were evaluated by comparing preoperative audiometric results with follow-up

28 data (at least 36 months after surgery). Logistic regression analyses were performed to

29 determine prognostic factors related to long-term hearing success. These factors

30 included classification and stage of cholesteatoma, stapes condition, ossicular chain

31 damage, active infection of the middle ear, state of the contralateral ear, preoperative

32 hearing thresholds, gender, and age. Results: The mean follow-up period was 49.2

33 months. The recidivism rate was $6 \%$ (4 of 66 ears). The pure-tone average significantly

34 improved from $50.78 \pm 19.98$ to $40.81 \pm 21.22 \mathrm{~dB}$ hearing level $(\mathrm{HL} ; \mathrm{p}<0.001)$. Air-

35 bone gaps significantly improved from $26.26 \pm 10.53$ to $17.58 \pm 8.21 \mathrm{~dB} H \mathrm{HL}(\mathrm{p}<0.001)$.

36 In multivariate logistic regression analysis, early-stage disease $(p=0.021)$ and pars 
37 flaccida cholesteatoma $(p=0.036)$ exhibited statistically significant correlations with

38 successful hearing preservation.

39 Conclusion: Functional cholesteatoma surgery with autologous bone grafts

40 reconstruction is an effective approach to significantly improve hearing with low

41 recidivism rates. Localized disease and pars flaccida cholesteatoma were two

42 independent predictors of successful hearing preservation.

\section{Introduction}

45 Surgical removal of cholesteatoma is the standard treatment once the diagnosis is

46 established ${ }^{1}$. However, cholesteatoma surgery is particularly challenging because the

47 goals of removing the complete cholesteatoma and maintaining the normal functions of

48 the temporal bone appear to conflict. Canal wall down (CWD) and canal wall up (CWU)

49 mastoidectomies are the two main surgical techniques conventionally used for the

50 management of middle ear cholesteatoma. ${ }^{2}$. Compared with CWD mastoidectomy, CWU

51 mastoidectomy results in more favorable hearing outcomes and prevents cavity

52 problems. ${ }^{3}$ However, studies have reported higher rates of recidivism that may be

53 attributable to insufficient exposure in CWU mastoidectomy ${ }^{3-5}$. A functional

54 cholesteatoma surgical technique combining the advantages of both CWU and CWD 
55 mastoidectomies has been developed. Different names have been used for this

56 developed technique including flexible endaural approach ${ }^{6,7}$, tailor-made mastoidectomy

578 , retrograde mastoidectomy ${ }^{9-11}$, the inside-out approach ${ }^{12}$, and limited mastoidectomy

58 13. Although differences exist among these various procedures, their main principle

59 remains the same-tracking lesions to their ends. In this procedure, uninvolved middle

60 ear cleft structures and the ear's normal function are preserved. Acceptable recidivism

61 rates have been reported for functional mastoidectomy ${ }^{14,15}$ compared with CWU and

62 CWD mastoidectomies ${ }^{16}$.

64 Mastoid obliteration and canal wall reconstruction, followed by disease removal, are

65 mandatory to prevent leaving a cavity. ${ }^{17}$ Various materials have been used for mastoid

66 obliteration including a bone plate ${ }^{11}$, aural cartilage ${ }^{15}$, and fat ${ }^{18}$. In this study, we used

67 autologous bone grafts as the primary material for reconstruction. Studies examining

68 the long-term follow-up outcomes of the application of autologous bone grafts for

69 reconstructing tympanomastoid defects in functional cholesteatoma surgery are lacking.

70 Therefore, the present study evaluated long-term hearing outcomes of functional

71 mastoidectomy in which autologous bone grafts were used as the primary material for

72 reconstruction. In addition, this study analyzed factors that can predict successful 
73 hearing preservation.

74

75 Materials and methods

76 This retrospective study evaluated patients with acquired middle ear cholesteatoma who

77 were admitted for functional tympanomastoidectomy in a single tertiary hospital between

782010 and 2018 with a minimum follow-up of 3 years. A total of 116 patients were assessed

79 for eligibility, and of these 116 patients, 50 were excluded. Finally, 66 patients (66 ears)

80 aged between 18 and 74 years were enrolled in this study. All of the surgeries were

81 performed by a single surgeon. Exclusion criteria were as follows: (1) unavailability of

82 complete audiometry data or follow-up hearing examination data for less than 3 years

83 postoperatively, (2) revised surgery, (3) presence of congenital cholesteatoma, (4)

84 diagnosis other than cholesteatoma, (5) undergoing a procedure other than functional

85 tympanomastoidectomy, and (6) cholesteatoma of the external auditory canal. The study

86 flow diagram is shown in Fig.1. This study was approved by the Institutional Review Board

87 of China Medical University Hospital (project approval number CMUH110-REC3-004). All

88 methods were carried out in accordance with relevant guidelines and regulations. This

89 study involved no prospectively collected data so there was no access to patients or opportunity to seek informed consent. Consent for waiver of consent from Institutional 
91 Review Board was obtained.

92

93 The following detailed information was collected for each patient: age, sex, affected ear,

94 follow-up duration, types of tympanoplasty, group and stage of cholesteatoma, severity

95 of ossicle destruction, presence of the stapes suprastructure, state of the contralateral

96 ear, and infection at the time of surgery (Table 1). The group and stage of

97 cholesteatoma were determined according to the classification and staging system

98 proposed by the European Academy of Otology and Neurotology/Japan Otological

99 Society (EAONO/JOS) Joint Committee ${ }^{19}$. In this study, patients were classified into

100 two main groups: (1) pars flaccida and (2) pars tensa or combination of pars flaccida

101 and pars tensa. The STAM system, which is used for staging cholesteatoma, divides

102 the middle ear cavity into four sites: difficult access sites (S), tympanic cavity (T), attic

103 (A), and mastoid (M). The difficult access sites (S) include S1, the supratubal recess

104 (also called the anterior epitympanum or protympanum), S2, and the sinus tympani.

105 Stage I indicates that the cholesteatoma is localized in the primary site-the attic (A) for

106 the pars flaccida group and the tympanic cavity $(T)$ for the pars tensa group. Stage II

107 indicates that the cholesteatoma involves two or more sites. Stage III indicates the

108 findings of extracranial complications or pathologic conditions including facial palsy, 
109 labyrinthine fistula, labyrinthitis, postauricular abscess or fistula, zygomatic abscess,

110 neck abscess, canal wall destruction (more than half the length of the bony ear canal),

111 tegmen destruction, and adhesive otitis. Stage IV indicates the presence of

112 cholesteatoma along with intracranial complications including bacterial meningitis,

113 epidural abscess, subdural abscess, cerebral abscess, sinus thrombosis, and brain

114 herniation.

115

116 Audiometric data were recorded at the time of admission before treatment and every 3

117 months after surgery in the first year and then every 6 months in the second year.

118 Patients who did not undergo follow-up hearing tests for more than 3 years were

119 excluded. During the treatment, hearing changes were examined by comparing hearing

120 test results obtained before the treatment with those obtained at the latest follow-up.

121 Pure-tone thresholds for air and bone conduction were conducted at the frequencies of

$1220.25,0.5,1,2,4$, and $8 \mathrm{kHz}$. The pure-tone average (PTA) was determined by

123 calculating the mean of the $0.5,1,2$, and $4 \mathrm{kHz}$ thresholds of air conduction. Air-bone

124 gaps (ABGs) were calculated on the basis of air and bone conduction results obtained

125 at the thresholds of $0.5,1,2$, and $4 \mathrm{kHz}$. The mean preoperative and postoperative PTA

126 and ABGs as well as improvements in the PTA and ABGs were recorded. 
128 In this study, meeting the following criteria indicated successful hearing preservation

129 after functional tympanomastoidectomy: (1) no recurrent or residual cholesteatoma

130 during the follow-up period and (2) gain of at least $15 \mathrm{~dB}$ in the PTA when compared

131 with preoperative data or conservation of the hearing value in patients with normal

132 preoperative hearing (PTA $\leq 25 \mathrm{~dB})$. Cholesteatoma recidivism was suspected via

133 otoscope examination and deteriorated audiometry during follow-up period, and was

134 established following revised surgery. The success rate of the hearing conservation was

135 analyzed using the criteria suggested by the Japan Clinical Otology Committee ${ }^{20}$.

136 According to these criteria, 33 and 33 patients were included in the successful hearing

137 group and unsuccessful hearing group, respectively.

139 To determine the factors that predict successful hearing preservation, the following

140 variables were analyzed: age at surgery ( $\leq 45$ years or $>45$ years), sex (female or male),

141 preoperative PTA ( $\leq 40 \mathrm{~dB}$ or $>40 \mathrm{~dB}$ ), cholesteatoma groups (pars flaccida, pars tensa,

142 or a combination of pars flaccida and pars tensa), cholesteatoma stage (stage I or stage

143 II-IV), active infection at the time of surgery (absence or presence of otorrhea),

144 ossicular chain destruction (normal or destruction of one, two, or three ossicles), stapes 
145 condition (presence or absence of the suprastructure), and state of the contralateral ear

146 (normal or otitis media with effusion/atelectasis/eardrum perforation). One study

147 evaluated the influence of the age factor on the prognosis for tympanoplasty revealed

148 that the group aged under 45 years had a trend for higher hearing success, so we

149 decided on 45 years as a criterion for two groups of patients ${ }^{21}$. We put both patients

150 with intact ossicular chain and with one ossicle destroyed in one group based on the

151 Cholesteatoma-Atelectasis-Ossicle staging system of cholesteatoma ${ }^{22}$.

152

153 Functional tympanomastoidectomy was performed similar to the procedure reported in

154 previous studies ${ }^{9,23}$. Under general anesthesia, a postauricular incision was made to

155 expose the temporalis fascia, which was used for grafting. An adequate amount of the

156 temporalis fascia was harvested to perform eardrum and external auditory canal (EAC)

157 reconstruction later, including tympanoplasty and the coverage of bone grafts used for

158 EAC reconstruction. A periosteal incision was made near the opening of the EAC but

159 without penetration into the EAC lumen, followed by the elevation of the periosteum into

160 the lateral ear canal. After identifying Henle's spine, elevation of the posterior meatal

161 epithelium continued until reaching $1 \mathrm{~cm}$ deep relative to Henle's spine. A U-shaped

162 incision was made over the elevated posterior meatal wall, and the vascular strip was 
163 then turned outside. A rubber Penrose drain was inserted to retract the auricle and

164 lateral canal anteriorly. Weitlaner and Fisch articulated retractors (Karl Storz, Tuttlingen,

165 Germany) were applied to gain further exposure. Bone grafts were harvested from the

166 healthy mastoid cortex using a mastoid chisel and metal mallet (Karl Storz, Tuttlingen,

167 Germany) and soaked in ofloxacin solution (Fig. 2A). After denuding around the

168 perforation (if present), the tympanomeatal flap was raised. Canaloplasty and scutum

169 removal were performed to gain access to the cholesteatoma. The extent of functional

170 tympanomastoidectomy was dependent on how far the cholesteatoma had expanded,

171 variously requiring atticotomy, atticoantrotomy, or atticoantromastoidectomy (Fig. 2B).

172 After complete removal of the cholesteatoma epithelium, the open mastoid cavity, aditus

173 ad antrum, and epitympanum were filled with autologous bone grafts for reconstructing

174 and maintaining the anatomy of the EAC (Fig. 2C-D). Temporalis fascia grafts were

175 applied in an underlay fashion to cover the bone grafts and patch any eardrum defect. A

176 superiorly based temporalis muscle flap was used to prevent cavity problems if the

177 harvested bone grafts and fascial graft were inadequate. Tegmen dehiscence with dura

178 exposure was covered and repaired using a pedicled flap. A smooth surface without any

179 defects over the entire EAC was ensured after reconstruction. 
181 Tympanoplasty was performed as a single-stage procedure in all cases after the

182 removal of cholesteatoma by using functional tympanomastoidectomy. Patients in this

183 study underwent three types of tympanoplasty: type I, type III (Fig. 2C-D), and type IV.

184 Ossiculoplasty was performed in patients whose ossicular chain was destroyed by the

185 cholesteatoma or removed for better exposure. Type III tympanoplasties performed in

186 patients could be subdivided according to the ossiculoplasty technique used: (1) minor

187 columella subtype (type III-m): the autologous incus, malleus, or cortical bone were

188 harvested and sculptured as the columella between the stapes head and tympanic

189 membrane and (2) major columella subtype (type III-M): an autologous bone graft or

190 prosthesis was set between the stapes footplate and tympanic membrane. In type IV

191 tympanoplasty, the fascia graft was set upon the mobile stapes footplate with a shielded

192 round window ${ }^{24}$.

193

194 Statistical analyses were performed using SPSS version 24.0 (IBM Corp., Armonk, NY,

195 USA). A paired-samples $t$ test was performed to compare numerical variables and

196 determine significant differences between preoperative and postoperative hearing

197 results. One-way analysis of variance and Fisher's exact test were used to compare

198 hearing gains and the rate of successful hearing preservation, respectively, among 
199 different tympanoplasty types. Univariate and multivariate logistic regression analyses

200 were performed to determine factors that were related to long-term hearing success. A

$201 p$ value of $<0.05$ was considered statistically significant.

202

203 Results

204 The mean age of patients was $46.5 \pm 14.5$ years (range: $8-74$ years). Among 66

205 patients (66 ears), 28 were aged $<45$ years. Of 66 patients, $30(45.5 \%)$ were men. The

206 left and right ears were involved in 33 and 33 patients, respectively. Among 66 patients,

$20746(69.7 \%)$ presented with more than mild hearing loss preoperatively (PTA $>40 \mathrm{~dB}$ ).

208 The mean follow-up period was $49.2 \pm 21.6$ months (range: $36-114$ months). Type I

209 tympanoplasty was conducted in $20(30.3 \%)$ of 66 ears. Type III tympanoplasty with the

210 minor columella and type III tympanoplasty with the major columella were performed in

$21137(56.1 \%)$ of 66 ears and $7(10.6 \%)$ of 66 ears, respectively. Type IV tympanoplasty

212 was performed in $2(3 \%)$ of 66 ears. All patients were classified as pars flaccida, pars

213 tensa, or a combination of pars flaccida and pars tensa according to tympanic

214 membrane status. Of the 66 ears with cholesteatoma, 59 (89.4\%), 5 (7.6\%), and 2

$215(3.0 \%)$ were included in the groups of pars flaccida, pars tensa, and a combination of

216 both pars flaccida and pars tensa, respectively. In this study, cases of pars tensa and 
217 the combined type were classified into the pars tensa group. A total of 29 (43.9\%), 33

$218(50.0 \%), 4(6.1 \%)$, and $0(0 \%)$ patients had stage I, stage II, stage III, and stage IV

219 disease, respectively. Of four patients with stage III disease, three had labyrinthine

220 fistulas and one had delayed facial palsy. The contralateral ear was normal in 40

$221(60.6 \%)$ of 66 patients, and otitis media with effusion/atelectasis/ eardrum perforation

222 was found in $26(39.4 \%)$ of 66 patients. The ossicular chain status was confirmed

223 surgically. A total of $27(40.9 \%), 28(42.4 \%)$, and $11(16.7 \%)$ patients had normal

224 ossicles or destruction in one ossicle, destruction in two ossicles, and destruction in

225 three ossicles, respectively. Furthermore, $16(24.2 \%)$ patients presented with active ear

226 infection at the time of surgery, manifesting as otorrhea. Destruction of the stapes

227 suprastructure was observed in $9(13.6 \%)$ of 66 patients, and remained stapes integrity

228 was noted in $57(86.4 \%)$ patients. According to the criteria used to determine successful

229 hearing preservation in this study, $33(50.0 \%)$ patients were included in the successful

230 hearing group and $33(50.0 \%)$ patients were included in the unsuccessful hearing

231 group.

232

233 Long-term hearing outcomes in patients who underwent surgery were evaluated by

234 comparing the preoperative PTA and ABGs with postoperative results. The PTA (mean 
$235 \pm$ standard deviation [SD]) significantly improved from $50.78 \pm 19.98 \mathrm{~dB}$ hearing level

$236(\mathrm{HL})$ to $40.81 \pm 21.22 \mathrm{~dB} \mathrm{HL}$ (mean difference, $9.96 \pm 13.73 \mathrm{~dB} \mathrm{HL} ; \mathrm{p}<0.001$ ). ABGs

237 (mean \pm SD) significantly improved from $26.26 \pm 10.53 \mathrm{~dB} \mathrm{HL}$ to $17.58 \pm 8.21 \mathrm{~dB} \mathrm{HL}$

238 (mean difference: $8.48 \pm 10.27 \mathrm{~dB} \mathrm{HL} ; \mathrm{p}<0.001$; Table 2). The improvement in both

239 PTA and ABGs demonstrated significant overall hearing recovery after functional

240 mastoidectomy in patients with cholesteatoma.

241

242 The hearing outcomes of different tympanoplasty types were evaluated on the basis of

243 the hearing gains of the PTA and ABGs. Improvements in the PTA (mean \pm SD) were

$2449.06 \pm 11.52,9.86 \pm 13.35,13.39 \pm 23.03$, and $8.75 \pm 13.73 \mathrm{~dB}$ HL in type I, type III-m,

245 type III-M, and type IV tympanoplasties, respectively. No significant differences in the

246 improvement of the PTA were observed among different tympanoplasty types $(p=$

247 0.914). Postoperative gains in ABGs (mean \pm SD) were $6.44 \pm 9.86,9.43 \pm 10.41,10.54$

$248 \pm 12.46$, and $4.38 \pm 0.88 \mathrm{~dB} \mathrm{HL}$ in type I, type III-m, type III-M, and type IV

249 tympanoplasties, respectively. No significant differences in postoperative gains in ABGs

250 were noted among different tympanoplasty types $(p=0.646)$. The preoperative PTA

251 was compared between different tympanoplasty types to validate the reliability of the

252 aforementioned results. A statistically significant difference in the baseline PTA was 
253 noted $(p=0.003)$, and the post hoc test results indicated that patients who received

254 type III-M tympanoplasty had poorer preoperative hearing performance than did those

255 who received type I tympanoplasty. The rates of successful hearing preservation were

$25655.0 \%, 51.4 \%, 28.6 \%$, and 50.0\% in type I, type III-m, type III-M, and type IV

257 tympanoplasties, respectively. No significant differences in the rate of successful

258 hearing preservation were observed between different tympanoplasty types $(p=0.788$;

259 Table 3).

260

261 Logistic regression analysis was performed to predict factors that are related to long-

262 term hearing success, and the results are listed in Table 4. The results of univariate

263 analysis demonstrated that the likelihood of successful hearing preservation was

264 significantly related to the early stage of cholesteatoma (odds ratio [OR] $=3.12 ; 95 \%$

265 confidence interval $[\mathrm{Cl}]=1.13-8.60 ; p=0.028)$. There was a trend toward higher

266 probability of successful hearing preservation in patients with pars flaccida

267 cholesteatoma $(\mathrm{OR}=7.11,95 \% \mathrm{Cl}=0.81-62.79, \mathrm{p}=0.078)$ and those without otorrhea

268 at the time of surgery $(\mathrm{OR}=2.80,95 \% \mathrm{Cl}=0.85-9.26, \mathrm{p}=0.091)$. Hearing success

269 was not predicted by age $(p=0.619)$, sex $(p=0.324)$, preoperative PTA $(p=0.593)$,

270 ossicular chain destruction $(p=0.389)$, integrity of the stapes $(p=0.290)$, and state of 
271 the contralateral ear $(p=.315)$. The findings of multivariate logistic regression indicated

272 that early-stage disease $(\mathrm{OR}=4.41,95 \% \mathrm{Cl}=1.26-15.49, \mathrm{p}=0.021)$ and pars flaccida

273 cholesteatoma $(O R=13.55,95 \% \mathrm{Cl}=1.19-154.68, \mathrm{p}=0.036)$ were significantly

274 correlated with successful hearing preservation. Other variables were not found to be

275 related to hearing success in multivariate analysis.

276

277 The rate of postoperative complications was $13.6 \%$ (9 of 66 ears). Delayed facial palsy

278 was noted in one patient (1.5\%) on postoperative day 7 , but this patient exhibited

279 complete recovery 1 month postoperatively. Two (3\%) patients developed dry eardrum

280 perforation, and two (3\%) patient developed otitis media with effusion during the follow-

281 up period. Four patients (6\%) experienced recidivism during the follow-up period: three

282 patients (4.5\%) developed recurrence, and one patient $(1.5 \%)$ had residual disease.

283 The sites of recurrence in the three patients were all in the epitympanum region. A

284 residual keratin pearl in the external auditory canal (EAC) was found in one patient. All

285 four patients who experienced recidivism received revised surgery and recovered well

286 without any new adverse events. Accumulation of keratin debris and purulent discharge

287 in EAC were frequently noted during preoperative otoscope evaluation (Fig. 3A). At 12

288 months after surgery, $48(72.7 \%)$ and $18(27.3 \%)$ patients exhibited a healed and dry 
289 EAC (Fig. 3B) and a healed EAC with accumulated ear wax, respectively. High-

290 resolution computed tomography (CT) of temporal bone were performed in all patients

291 for preoperative evaluation (Fig. 4A). CT were also completed in all patients more than

292 three years postoperatively. Postoperative CT scans revealed that the autologous bone

293 grafts use for EAC reconstruction is well maintained during long-term follow-up (Fig.

294 4B). Persistent otorrhea related to granulation formation, poor graft uptake, and

295 otomycosis was noted in four (6.0\%) patients. All patients with significant ear discharge

296 responded well to a topical ear solution and in-office local treatment.

297

298 Discussion

299 The results of this study revealed that patients with acquired cholesteatoma presented

300 significant overall hearing recovery with satisfactory recidivism occurrence rates after

301 undergoing functional tympanomastoidectomy involving the use of autologous bone

302 grafts as the primary material for reconstruction.

303

304 The use of the inside-out technique with the removal of the posterior auditory canal wall

305 for exposure and eradication of cholesteatoma, followed by the reconstruction of the

306 open cavity, has been proposed in several studies ${ }^{25-29}$. However, a flawless strategy for 
307 the management of the canal wall defect after cholesteatoma removal is not yet

308 available. ${ }^{30}$

309

310 In their retrospective study conducted in 2004, Dornhoffer evaluated the long-term

311 results of retrograde mastoidectomy, followed by the reconstruction of the auditory

312 canal wall defect using the cymba cartilage. ${ }^{15}$ The harvested cymba cartilage in the

313 fitting curvature was placed in the grooves for reconstruction. However, appropriate

314 fitting of the cymba cartilage is challenging with an absolute learning curve. Abougabal

315 et al. reported the surgical outcomes of retrograde mastoidectomy with canal wall

316 reconstruction that involved the use of autograft bone plates that were secured in

317 appropriate positions by fitting the graft in created grooves. ${ }^{11}$ Glass ionomer cement and

318 cartilage pieces were used to stabilize the bone graft. Nonetheless, fitting and fixation of

319 the bone plate were still challenging.

320

321 To the best of our knowledge, few studies have examined the long-term treatment

322 efficacy and safety of applying autologous bone grafts as the primary material for

323 reconstructing tympanomastoid defects in functional cholesteatoma surgery. Our

324 retrospective study results showed that functional mastoidectomy followed by canal wall 
325 reconstruction with bone grafts significantly improved hearing and led to a low

326 recidivism rate. An ideal surgical procedure should be quick and simple. Using bone

327 grafts harvested from the healthy mastoid cortex for EAC defect reconstruction and

328 mastoid obliteration without additional materials can improve operational efficiency.

330 According to audiometric outcomes, the PTA and ABGs demonstrated statistically

331 significant improvements. This finding confirmed that this type of operation could yield

332 reliable surgical results with significant recovery and preservation of hearing. The

333 hearing outcomes of patients included in this study were comparable to those in

334 previous studies on cholesteatoma surgery with canal wall reconstruction. Kuo et al.

335 reported the long-term hearing results and effects of mastoid obliteration/exclusion

336 using bone chips as the sole material for reconstruction in patients with cholesteatoma

337 receiving retrograde tympanomastoidectomy. ${ }^{23}$ Significant gains in both PTA (mean

338 difference, $5.7 \mathrm{~dB} ; \mathrm{p}<0.001$ ) and ABGs (mean difference, $6.96 \mathrm{~dB} ; \mathrm{p}<0.001$ ) were

339 noted. In their retrospective study, Haeusler et al. reported that 586 patients (604 ears)

340 received inside-out cholesteatoma surgery with cartilaginous reconstruction of the canal

341 wall. ${ }^{12}$ A significant improvement in hearing was reported with a mean $A B G$ of $<30 \mathrm{~dB}$ in

$34278 \%$ patients postoperatively, and ABGs improved by an average of $12 \mathrm{~dB}$. 
344 Different tympanoplasty types exerted no significant effect on postoperative hearing

345 gains and successful hearing preservation rates in our study. Patients who underwent

346 type IV tympanoplasty tended to demonstrate poor hearing recovery with lower PTA

347 and ABG gains. However, only two patients received type IV tympanoplasty, and the

348 small sample size might have increased the margin of error. The integrity of the stapes

349 was considered to be a crucial factor that predicts postoperative hearing outcomes. An

350 intact stapes structure with a mobile foot plate (type I, II, and III tympanoplasty) showed

351 a significant correlation with postoperative ABGs of $30 \mathrm{~dB}$ or lower in more than $80 \%$ of

352 patients in one study; by contrast, this correlation was observed in only $70 \%$ of patients

353 who underwent type IV tympanoplasty ${ }^{12}$. No significant difference was observed in

354 hearing outcomes between type III-m and type III-M tympanoplasties in our study. A

355 study comparing type III-m with partial ossicular replacement prosthesis (PORP) and

356 type III-M tympanoplasty with total ossicular replacement prosthesis (TORP) reported

357 that both types of reconstruction showed no difference in hearing outcomes ${ }^{15}$. Hence,

358 restoration of ossicular coupling using either the autograft columella, PORP, or TORP

359 could lead to satisfactory hearing results postoperatively, in contrast to type IV

360 tympanoplasty with solely acoustic coupling ${ }^{31}$. 
362 Patients and surgeons should know possible predictive factors related to successful

363 hearing preservation after surgery. Multiple variables were included in univariate and

364 multivariate logistic regression analyses. Early-stage cholesteatoma and pars flaccida

365 cholesteatoma were determined to be independent predictors of hearing success in the

366 multivariate analysis. This finding is consistent with that of a study conducted in 2020 by

367 Ardiç et al. ${ }^{32}$. The authors evaluated the surgical outcomes of cholesteatoma surgery

368 and correlated them with the classifications of the newly proposed EAONO/JOS staging

369 system. A significant correlation was observed between the classification of

370 cholesteatoma and hearing results, and patients with pars flaccida retraction pocket

371 cholesteatoma were observed to have the most favorable hearing outcomes.

372 Furthermore, in all cholesteatoma groups, higher-stage disease was related to poor

373 hearing results. Surgical techniques in the study included transcanal atticotomy and

374 CWD and CWU mastoidectomies. Fukuda et al. investigated prognostic factors for

375 hearing outcomes in patients with pars flaccida cholesteatoma according to the

376 EAONO/JOS system ${ }^{33}$. They noted favorable hearing outcomes in patients with early-

377 stage cholesteatoma. However, different techniques including CWD and CWU

378 mastoidectomies were used in the study. This finding suggests that an inconsistent 
379 correlation might exist between various surgical methods using the same staging

380 system. Our study utilized the EAONO/JOS staging system for patients who received

381 only functional cholesteatoma surgery; thus, its findings can be useful and reliable in the

382 same group of patients. In our study, the preoperative hearing level was not a significant

383 predictor. This finding is not consistent with those of previous studies. Kuo et al.

384 reported that preoperative serviceable hearing was not a significant favorable predictor

385 in multivariate analysis ${ }^{22}$. Chadha et al. reported that air-conduction (AC) thresholds

386 were increased by preoperative AC thresholds in patients who underwent CWU and

387 CWD surgery ${ }^{4}$. Inconsistent results among studies might be attributable to different

388 surgical techniques. The stapes integrity has been considered to be a crucial predictor

389 of successful hearing outcomes ${ }^{12,22}$. An intact stapes with a mobile foot plate was

390 related to a higher probability of hearing success. The small sample size of the group

391 with an absent stapes suprastructure might have led to nonsignificant results in our

392 study. Risk factors such as ossicular chain damage, active infection of the middle ear,

393 and contralateral ear disease were significant predictors of postoperative hearing

394 outcomes reported in previous studies ${ }^{34-36}$. However, our findings did not support these

395 results. With the improvement of surgical techniques and perioperative care, these risk

396 factors might have less effect on hearing success. 
398 The rate of recidivism in this study was $6 \%$ (4 of 66 ears): three (4.5\%) patients

399 developed recurrence and one (1.5\%) patient had residual disease. A recent meta-

400 analysis reported that the recidivism rate ranged from $5 \%$ to $17 \%$ in patients who

401 underwent CWD mastoidectomy and $9 \%$ to $70 \%$ in patients who underwent CWU

402 mastoidectomy ${ }^{16}$. According to our results, functional tympanomastoidectomy involving

403 the use of bone grafts for reconstruction not only could achieve satisfactory hearing

404 preservation but also resulted in a lower rate of recidivism that was comparable to that

405 of CWD mastoidectomy. Dornhoffer et al. reported that the recurrence and residual

406 disease were $16 \%$ ( 8 of 50 ears) and $4 \%$ ( 2 of 50 ears), respectively, in patients

407 receiving retrograde mastoidectomy with cartilaginous canal wall defect reconstruction

408 15. Comparable surgical outcomes were also noted in a study in which patients

409 underwent similar surgical procedures as patients in this study: $2.9 \%$ (3 of 102) of

410 patients developed recurrence and 1\% (1 of 102) of patients had residual disease ${ }^{23}$. All

411 three cases of recurrence in our study were in the epitympanum region. Obliteration of

412 the epitympanum or epitympanoplasty has been suggested to prevent the formation of a

413 retraction pocket and to reduce the recurrence rate ${ }^{37}$. The occurrence of recurrent

414 disease in our study may be related to the incomplete exposure of the cholesteatoma 
415 matrix or late necrosis of the bone graft.

416

417 The major limitation of this study is the lack of comparison groups. Second, the

418 retrospective analysis performed in this study may be a potential source of selection

419 bias. Third, making comparisons among studies is difficult when evaluating hearing-

420 related prognostic factors because no standard definition of successful hearing

421 preservation is available. In the future, prospective trials that incorporate a standard

422 definition of hearing recovery and a unified cholesteatoma staging system are needed.

423 Studies examining the long-term outcomes of functional tympanomastoidectomy using

424 primary autologous bone grafts for reconstruction are warranted because recurrence

425 rates are highly related to the length of follow-up.

426

427 Conclusion

428 Functional cholesteatoma surgery with the use of autologous bone grafts for

429 reconstruction could significantly improve hearing outcomes with low recidivism rates.

430 Early-stage disease and pars flaccida cholesteatoma were two independent positive

431 prognostic factors for successful hearing preservation. The combined use of staging

432 and classification may provide patients and surgeons with valuable information on 
hearing outcomes.

434

435 Acknowledgments

436 The authors would like to thank all colleagues of department of Otolaryngology Head

437 and Neck Surgery in China Medical University Hospital who provided insight and

438 expertise that greatly assisted the research. This manuscript was edited by Wallace

439 Academic Editing.

\section{References}

442 1. Kuo CL, Liao WH, Shiao AS. A review of current progress in acquired cholesteatoma 443 management. Eur Arch Otorhinolaryngol 2015;272:3601-3609.

444 2. Hellingman CA, Geerse S, de Wolf MJF, Ebbens FA, van Spronsen E. Canal wall up surgery 445 with mastoid and epitympanic obliteration in acquired cholesteatoma. Laryngoscope 446 2019;129:981-985.

447 3. Lucidi D, De Corso E, Paludetti G, Sergi B. Quality of life and functional results in canal wall 448 down vs canal wall up mastoidectomy. Acta Otorhinolaryngol Ital 2019;39:53-60.

449 4. Chadha NK, Jardine A, Owens D, Gillett S, Robinson PJ, Maw AR. A multivariate analysis of 450 the factors predicting hearing outcome after surgery for cholesteatoma in children. J Laryngol 451 Otol 2006;120:908-913.

452 5. Kerckhoffs KG, Kommer MB, van Strien TH, et al. The disease recurrence rate after the 453 canal wall up or canal wall down technique in adults. Laryngoscope 2016;126:980-987.

454 6. Alleva M, Paparella MM, Morris MS, da Costa SS. The flexible/intact-bridge 455 tympanomastoidectomy technique. Otolaryngol Clin North Am 1989;22:41-49.

456 7. Paparella MM, Jung TT. Intact-bridge tympanomastoidectomy. Otolaryngol Head Neck 457 Surg 1984;92:334-338.

458 8. Kuo CL SA, Liao WH, CF L. Functional surgery for pediatric cholesteatoma: a 25-year 459 longitudinal study. In: Takahashi $\mathrm{H}$ (ed) The 9th international conference on cholesteatoma and 
460 ear surgery, Nagasaki, Japan Cholesteatoma and ear surgery-an uptate 2012:225-227.

461 9. Dornhoffer JL. Retrograde mastoidectomy with canal wall reconstruction: a single-stage

462 technique for cholesteatoma removal. The Annals of Otology, Rhinology \& Laryngology

463 2000;109:1033-1039.

464 10. Minovi A, Venjacob J, Volkenstein S, Dornhoffer J, Dazert S. Functional results after

465 cholesteatoma surgery in an adult population using the retrograde mastoidectomy technique.

466 Eur Arch Otorhinolaryngol 2014;271:495-501.

467 11. Shewel Y, Abougabal A. Retrograde mastoidectomy with canal wall reconstruction with

468 bone graft in acquired cholesteatoma. The Egyptian Journal of Otolaryngology 2020;36.

469 12. Roth $\mathrm{TNaH}, \mathrm{R}$ Inside-Out Technique Cholesteatoma Surgery: A Retrospective Long-Term

470 Analysis of 604 Operated Ears Between 1992 and 2006. Otology \& Neurotology 2008;30:59-63.

471 13. Nikolopoulos TP, Gerbesiotis P. Surgical management of cholesteatoma: the two main

472 options and the third way--atticotomy/limited mastoidectomy. Int J Pediatr Otorhinolaryngol

473 2009;73:1222-1227.

474 14. Hatano M, Ito M, Yoshizaki T. Retrograde mastoidectomy on demand with soft-wall

475 reconstruction in pediatric cholesteatoma. Acta Otolaryngol 2010;130:1113-1118.

476 15. Dornhoffer JL. Retrograde Mastoidectomy with Canal Wall Reconstruction: A Follow-up

477 Report. Otology \& Neurotology 2004;25:653-660.

478 16. Tomlin J, Chang D, McCutcheon B, Harris J. Surgical technique and recurrence in

479 cholesteatoma: a meta-analysis. Audiol Neurootol 2013;18:135-142.

480 17. Harun A, Clark J, Semenov YR, Francis HW. The Role of Obliteration in the Achievement of

481 a Dry Mastoid Bowl. Otol Neurotol 2015;36:1510-1517.

482 18. Lee WS CJ, Song MH, Son EJ, Jung SH, Kim SH. Mastoid and Epitympanic Obliteration in

483 Canal Wall Up Mastoidectomy for Prevention of Retraction Pocket. Otology \& Neurotology

484 2005;26:1107-1111.

485 19. Yung M, Tono T, Olszewska E, et al. EAONO/JOS Joint Consensus Statements on the

486 Definitions, Classification and Staging of Middle Ear Cholesteatoma. J Int Adv Otol 2017;13:1-8.

487 20. Sasaki T, Xu A, Ishimoto S, Ito K, Yamasoba T, Kaga K. Results of hearing tests after total

488 middle ear reconstruction. Acta Otolaryngol 2007;127:474-479.

489 21. Tai CF, Ho KY, Juan KH. Age and the prognosis of tympanoplasty type I. The Kaohsiung

490 journal of medical sciences 1998;14:542-547.

491 22. Kuo CL, Shiao AS, Liao WH, Ho CY, Lien CF. Can long-term hearing preservation be expected

492 in children following cholesteatoma surgery? Results from a 14-year-long study of atticotomy-

493 limited mastoidectomy with cartilage reconstruction. Audiol Neurootol 2012;17:386-394.

494 23. Kuo CY, Huang BR, Chen HC, et al. Surgical results of retrograde mastoidectomy with

495 primary reconstruction of the ear canal and mastoid cavity. Biomed Res Int 2015;2015:1-12. 
496 24. Merkus P, Kemp P, Ziylan F, Yung M. Classifications of Mastoid and Middle Ear Surgery: A 497 Scoping Review. J Int Adv Otol 2018;14:227-232.

498 25. Roth TN, Ziglinas P, Haeusler R, Caversaccio MD. Cholesteatoma surgery in children: long499 term results of the inside-out technique. Int J Pediatr Otorhinolaryngol 2013;77:843-846.

500 26. Dornhoffer JL. Retrograde mastoidectomy. Otolaryngol Clin North Am 2006;39:1115-1127.

501 27. Kim BG, Kim HJ, Lee SJ, Lee E, Lee SA, Lee JD. Outcomes of Modified Canal Wall Down

502 Mastoidectomy and Mastoid Obliteration Using Autologous Materials. Clin Exp

503 Otorhinolaryngol 2019;12:360-366.

504 28. Skoulakis C, Koltsidopoulos P, Iyer A, Kontorinis G. Mastoid Obliteration with Synthetic

505 Materials: A Review of the Literature. The journal of international advanced otology

506 2019;15:400-404.

507 29. Lee HJ, Chao JR, Yeon YK, et al. Canal reconstruction and mastoid obliteration using

508 floating cartilages and musculoperiosteal flaps. The Laryngoscope 2017;127:1153-1160.

509 30. Mendlovic ML, Monroy Llaguno DA, Schobert Capetillo IH, Cisneros Lesser JC. Mastoid

510 obliteration and reconstruction techniques: A review of the literature. Journal of Otology

$5112021 ; 16: 178-184$.

512 31. Merchant SN, Ravicz, M. E. , Puria, S. , Voss, S. E. , Whittemore, K. R. , Peake, W. T. \&

513 Rosowski, J. J. . Analysis of Middle Ear Mechanics and Application to Diseased and

514 Reconstructed Ears. The American Journal of Otology 1997;18:139-154.

515 32. Ardic FN, Mengi E, Tumkaya F, Kara CO, Bir F. Correlation between Surgical Outcome and

516 Stage of Acquired Middle Ear Cholesteatoma: Revalidation of the EAONO/JOS Staging System. J

517 Int Adv Otol 2020;16:34-39.

518 33. Fukuda A, Morita S, Nakamaru Y, Hoshino K, Fujiwara K, Homma A. Short-Term Hearing

519 Prognosis of Ossiculoplasty in Pars Flaccida Cholesteatoma Using the EAONO/JOS Staging

520 System. J Int Adv Otol 2019;15:2-7.

521 34. Blom EF, Gunning MN, Kleinrensink NJ, et al. Influence of Ossicular Chain Damage on

522 Hearing After Chronic Otitis Media and Cholesteatoma Surgery: A Systematic Review and Meta-

523 analysis. JAMA Otolaryngol Head Neck Surg 2015;141:974-982.

524 35. Zwierz A, Haber K, Sinkiewicz A, Kalinczak-Gorna P, Tyra J, Mierzwinski J. The significance

525 of selected prognostic factors in pediatric tympanoplasty. Eur Arch Otorhinolaryngol

526 2019;276:323-333.

527 36. Salviz M, Bayram O, Bayram AA, et al. Prognostic factors in type I tympanoplasty. Auris 528 Nasus Larynx 2015;42:20-23.

529 37. Liu Y, Sun J, Zhao D, Lin Y. Epitympanoplasty with cartilage obliteration in the preservation

530 of posterior canal wall: a technique for surgical treatment of attic cholesteatoma. Eur Arch

531 Otorhinolaryngol 2014;271:939-946. 
Table $\mathbf{1}$ (on next page)

Demographics and characteristics of the patients 
Table 1. Demographics and characteristics of the patients $†$

\begin{tabular}{|c|c|}
\hline Variables & $n=66$ \\
\hline Age, years & $46.8 \pm 13.9(18-74)$ \\
\hline \multicolumn{2}{|l|}{ Gender } \\
\hline Female & $36(54.5)$ \\
\hline Male & $30(45.5)$ \\
\hline \multicolumn{2}{|l|}{ Side of ear } \\
\hline Left & $33(50.0)$ \\
\hline Right & $33(50.0)$ \\
\hline Follow-up periods, months & $49.2 \pm 21.6(36-114)$ \\
\hline \multicolumn{2}{|l|}{ Preoperative PTA } \\
\hline$\leqq 40 \mathrm{~dB}$ & $20(30.3)$ \\
\hline$>40 \mathrm{~dB}$ & $46(69.7)$ \\
\hline \multicolumn{2}{|l|}{ Type of tympanoplasty } \\
\hline Type I & $20(30.3)$ \\
\hline Type III-m & $37(56.1)$ \\
\hline Type III-M & $7(10.6)$ \\
\hline Type IV & $2(3.0)$ \\
\hline \multicolumn{2}{|l|}{ Group of cholesteatoma } \\
\hline Pars flaccida & $59(89.4)$ \\
\hline Pars tensa/ combined & $7(10.6)$ \\
\hline \multicolumn{2}{|l|}{ Stage of cholesteatoma } \\
\hline Stage I & $29(43.9)$ \\
\hline Stage II-IV & $37(56.1)$ \\
\hline \multicolumn{2}{|l|}{ Ossicles destruction } \\
\hline Normal or destruction of 1 ossicle & $27(40.9)$ \\
\hline Destruction of 2 ossicles & $28(42.4)$ \\
\hline Destruction of 3 ossicles & $11(16.7)$ \\
\hline \multicolumn{2}{|l|}{ Stapes } \\
\hline Present & $57(86.4)$ \\
\hline Absence of suprastructure & $9(13.6)$ \\
\hline \multicolumn{2}{|l|}{ Sate of contralateral ear } \\
\hline Normal & $40(60.6)$ \\
\hline OME/atelectasis/perforation & $26(39.4)$ \\
\hline \multicolumn{2}{|l|}{ Infection at the time of surgery } \\
\hline No otorrhea & $50(75.8)$ \\
\hline Otorrhea & $16(24.2)$ \\
\hline
\end{tabular}

$\mathrm{m}=$ minor columella; $\mathrm{M}=$ major columella; $\mathrm{OME}=$ Otitis media with effusion; $\dagger$ 
All values are presented as mean \pm standard deviation with ranges in parentheses or numbers with percentages in parentheses.

1 
Table 2 (on next page)

Surgical outcomes 
Table 2. Surgical outcomes $\dagger$

\begin{tabular}{lcc}
\hline \hline Audiometric results & $\mathrm{n}=66$ & $\mathrm{p}$ value \\
\hline Preoperative PTA (dB HL) & $50.78 \pm 19.98$ & \\
Postoperative PTA (dB HL) & $40.81 \pm 21.22$ & $<0.001^{*}$ \\
PTA improvement $(\mathrm{dB} \mathrm{HL})$ & $9.96 \pm 13.73$ & \\
\hline Preoperative ABG $(\mathrm{dB} \mathrm{HL})$ & $26.26 \pm 10.53$ & $<0.001^{*}$ \\
Postoperative ABG $(\mathrm{dB} \mathrm{HL})$ & $17.58 \pm 8.21$ & \\
ABG improvement $(\mathrm{dB} \mathrm{HL})$ & $8.48 \pm 10.27$ & \\
\hline \hline
\end{tabular}

PTA = pure-tone average; $A B G$ = air-bone gap

$\dagger$ All values are presented as mean \pm standard deviation

${ }^{*} p<0.05$ is considered statistically significant

1 


\section{Table $\mathbf{3}$ (on next page)}

Hearing gains and successful hearing preservation in different types of tympanoplasties 
Table 3. Hearing gains and successful hearing preservation in different types of tympanoplasties $†$

\begin{tabular}{|c|c|c|c|c|c|c|}
\hline & $\begin{array}{c}\text { Type I } \\
(\mathrm{n}=20)\end{array}$ & $\begin{array}{c}\text { Type III-m } \\
(\mathrm{n}=37) \\
\end{array}$ & $\begin{array}{c}\text { Type III-M } \\
(\mathrm{n}=7)\end{array}$ & $\begin{array}{l}\text { Type IV } \\
(\mathrm{n}=2) \\
\end{array}$ & & $p$ value* \\
\hline PTA gain & $9.06 \pm 11.52$ & $9.86 \pm 13.35$ & $13.39 \pm 23.03$ & $8.75 \pm 13.73$ & & 0.914 \\
\hline ABG gain & $6.44 \pm 9.86$ & $9.43 \pm 10.41$ & $10.54 \pm 12.46$ & $4.38 \pm 0.88$ & & 0.646 \\
\hline Preoperative PTA & $39.88 \pm 16.43$ & $52.47 \pm 19.92$ & $66.96 \pm 14.65$ & $71.88 \pm 6.19$ & 0.003 & $\begin{array}{c}\text { Post hoc: } \\
\text { Type III-M > Type I }\end{array}$ \\
\hline $\begin{array}{c}\text { Successful } \\
\text { hearing preservation }\end{array}$ & $11(55.0)$ & $19(51.4)$ & $2(28.6)$ & $1(50.0)$ & & 0.788 \\
\hline
\end{tabular}

PTA = pure-tone average; ABG = air-bone gap; Type III-m = minor columella; Type III-M = major columella

$\dagger$ All values are presented as mean \pm standard deviation with ranges in parentheses or numbers with percentages in parentheses.

* one-way ANOVA test for comparison of hearing gain and preoperative PTA; Fisher's exact test for rate of successful hearing preservation; $p<0.05$ is considered statistically significant 


\section{Table 4 (on next page)}

Logistic regression univariate and multivariate analyses for hearing success 


\begin{tabular}{|c|c|c|c|c|c|c|}
\hline Predictors & $\begin{array}{l}\text { Cases } \\
(n=66)\end{array}$ & $\begin{array}{c}\text { Success } \\
\mathrm{n}(\%)\end{array}$ & $\begin{array}{l}\text { Univariate } \\
\text { OR }(95 \% \mathrm{Cl})\end{array}$ & $\begin{array}{c}p \\
\text { value }\end{array}$ & $\begin{array}{l}\text { Multivariate } \\
\text { OR }(95 \% \mathrm{Cl})\end{array}$ & $\begin{array}{c}\mathrm{p} \\
\text { value }\end{array}$ \\
\hline \multicolumn{7}{|l|}{ Age } \\
\hline$\leqq 45$ years old & 28 & $13(46.4)$ & $0.78(0.29-2.08)$ & \multirow[t]{2}{*}{0.619} & $0.85(0.24-3.06)$ & \multirow[t]{2}{*}{0.808} \\
\hline$>45$ years old & 38 & $20(51.3)$ & 1 (ref) & & 1 (ref) & \\
\hline \multicolumn{7}{|l|}{ Gender } \\
\hline Female & 36 & $20(55.6)$ & $1.64(0.62-4.34)$ & \multirow[t]{2}{*}{0.324} & $1.13(0.33-3.87)$ & \multirow[t]{2}{*}{0.844} \\
\hline Male & 30 & $13(43.3)$ & 1 (ref) & & 1 (ref) & \\
\hline \multicolumn{7}{|l|}{ Preoperative PTA } \\
\hline$\leqq 40 \mathrm{~dB}$ & 20 & $11(55.0)$ & $1.33(0.47-3.83)$ & \multirow[t]{2}{*}{0.593} & $1.05(0.25-4.46)$ & \multirow[t]{2}{*}{0.943} \\
\hline$>40 \mathrm{~dB}$ & 46 & $22(47.8)$ & 1 (ref) & & 1 (ref) & \\
\hline \multicolumn{7}{|l|}{ Group of cholesteatoma } \\
\hline Pars flaccida & 59 & $32(54.2)$ & $7.11(0.81-62.79)$ & \multirow[t]{2}{*}{0.078} & $13.55(1.19-154.68)$ & \multirow[t]{2}{*}{$0.036^{*}$} \\
\hline Pars tensa/ combined & 7 & $1(14.3)$ & 1 (ref) & & 1 (ref) & \\
\hline \multicolumn{7}{|l|}{ Stage of cholesteatoma } \\
\hline Stage I & 29 & $19(65.5)$ & $3.12(1.13-8.60)$ & \multirow{2}{*}{$0.028^{*}$} & $4.41(1.26-15.49)$ & \multirow{2}{*}{$0.021^{*}$} \\
\hline Stage II-IV & 37 & $14(37.8)$ & 1 (ref) & & 1 (ref) & \\
\hline \multicolumn{7}{|l|}{ Infection at the time of surgery } \\
\hline No otorrhea & 50 & $28(56.0)$ & $2.80(0.85-9.26)$ & \multirow[t]{2}{*}{0.091} & $2.92(0.72-11.89)$ & \multirow[t]{2}{*}{0.135} \\
\hline Otorrhea & 16 & 5 (31.3) & 1 (ref) & & 1 (ref) & \\
\hline \multicolumn{7}{|l|}{ Ossicular chain } \\
\hline Normal or destruction of 1 ossicle & 27 & $14(51.9)$ & $1.89(0.45-7.97)$ & 0.389 & $1.01(0.08-12.17)$ & 0.993 \\
\hline Destruction of 2 ossicles & 28 & $15(53.6)$ & $2.02(0.48-8.49)$ & \multirow[t]{2}{*}{0.337} & $1.03(0.09-12.10)$ & \multirow[t]{2}{*}{0.979} \\
\hline Destruction of 3 ossicles & 11 & $4(36.4)$ & 1 (ref) & & 1 (ref) & \\
\hline \multicolumn{7}{|l|}{ Stapes } \\
\hline Present & 57 & $30(52.6)$ & $2.22(0.51-9.76)$ & \multirow[t]{2}{*}{0.290} & $1.24(0.09-16.52)$ & \multirow[t]{2}{*}{0.871} \\
\hline Absence of suprastructure & 9 & $3(33.3)$ & 1 (ref) & & & \\
\hline \multicolumn{7}{|l|}{ Sate of contralateral ear } \\
\hline Normal & 40 & $22(50.0)$ & $1.67(0.62-4.52)$ & \multirow[t]{2}{*}{0.315} & $1.05(0.31-3.62)$ & \multirow[t]{2}{*}{0.933} \\
\hline OME/atelectasis/perforation & 26 & $11(42.3)$ & 1 (ref) & & 1 (ref) & \\
\hline
\end{tabular}

PTA $(\mathrm{AC})=$ pure-tone average of air conduction threshold in $0.5 \mathrm{k}, 1 \mathrm{k}, 2 \mathrm{k}$ and $4 \mathrm{k} \mathrm{Hz}$; OME = otitis media with effusion

${ }^{*} p<0.05$ is considered statistically significant 
Figure 1

\section{The study flow diagram}

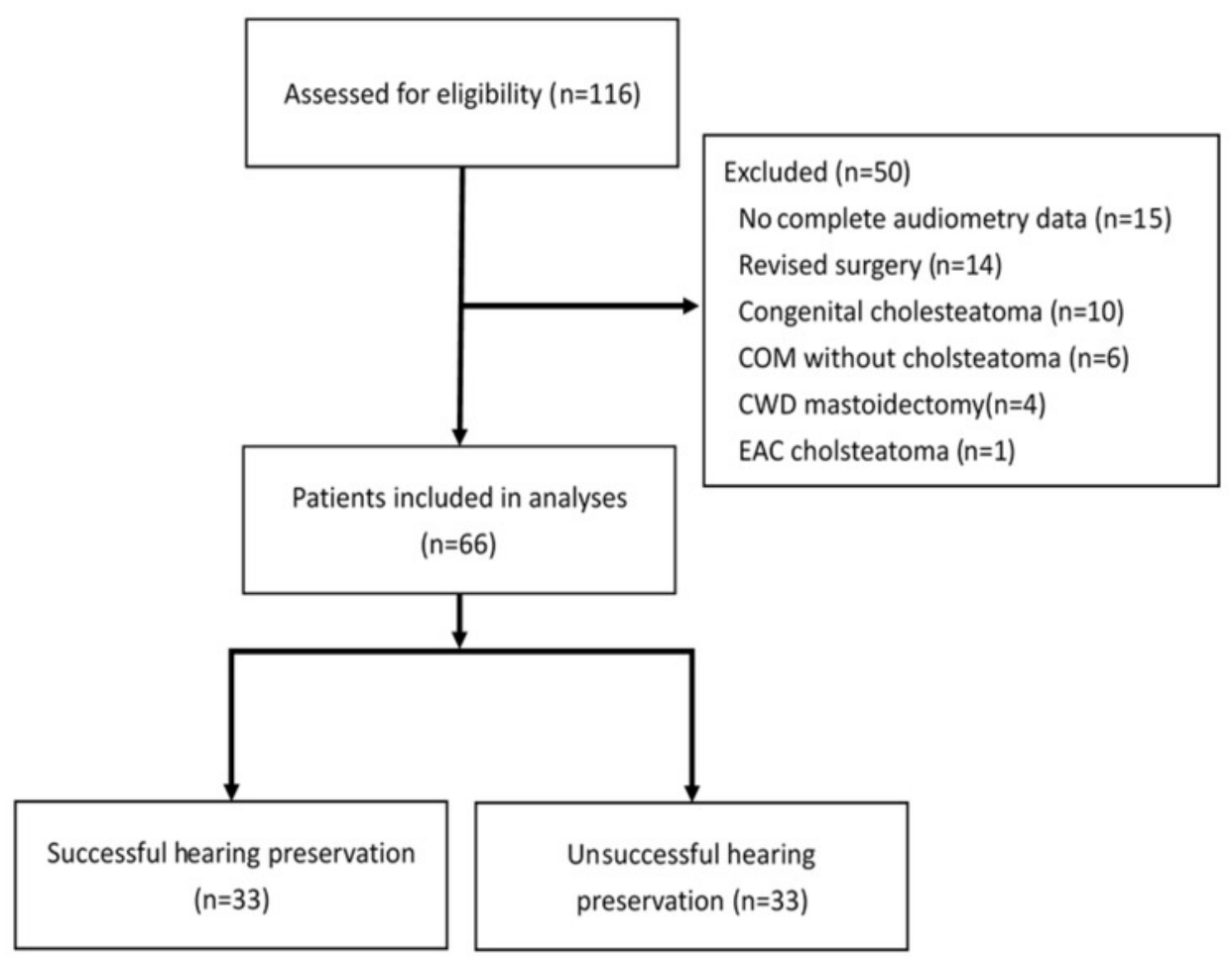




\section{Figure 2}

Intraoperative views

(A) Autologous bone grafts were harvested from the healthy mastoid cortex using a mastoid chisel. (B)Atticoantromastoidectomy was performed via inside-out approach to expose the cholesteatoma. (C) Type III tympanoplasty using cortical bone as columella was conducted in this case. (D) The open mastoid cavity, aditus ad antrum, and epitympanum were filled with bone grafts for reconstruction.
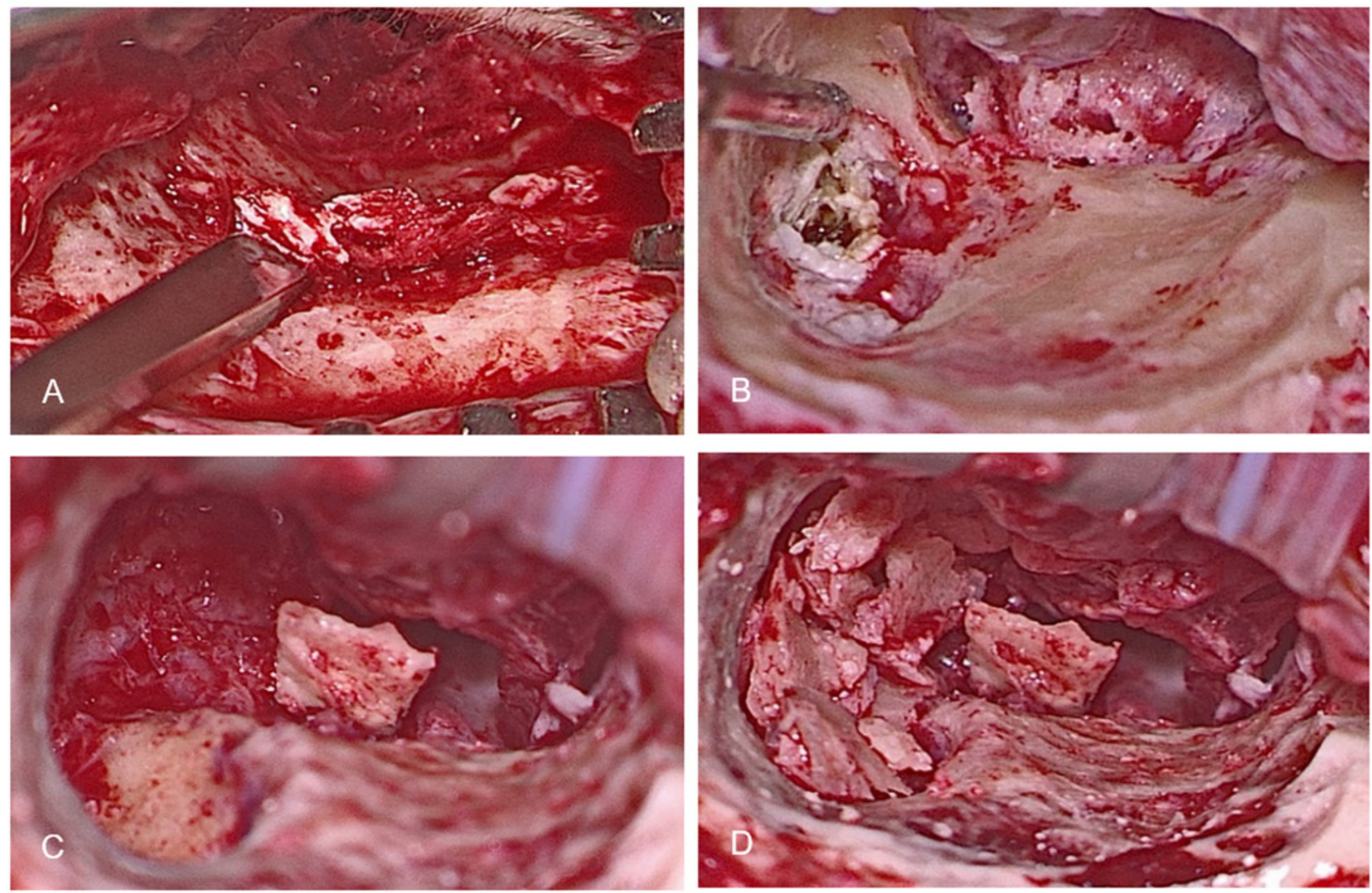


\section{Figure 3}

Preoperative and postoperative otoscope images

(A) Preoperative otoscope image showed keratin accumulates within the attic retraction pocket. (B) At 12 months after surgery, healed and dry external auditory canal and intact eardrum can be noted.
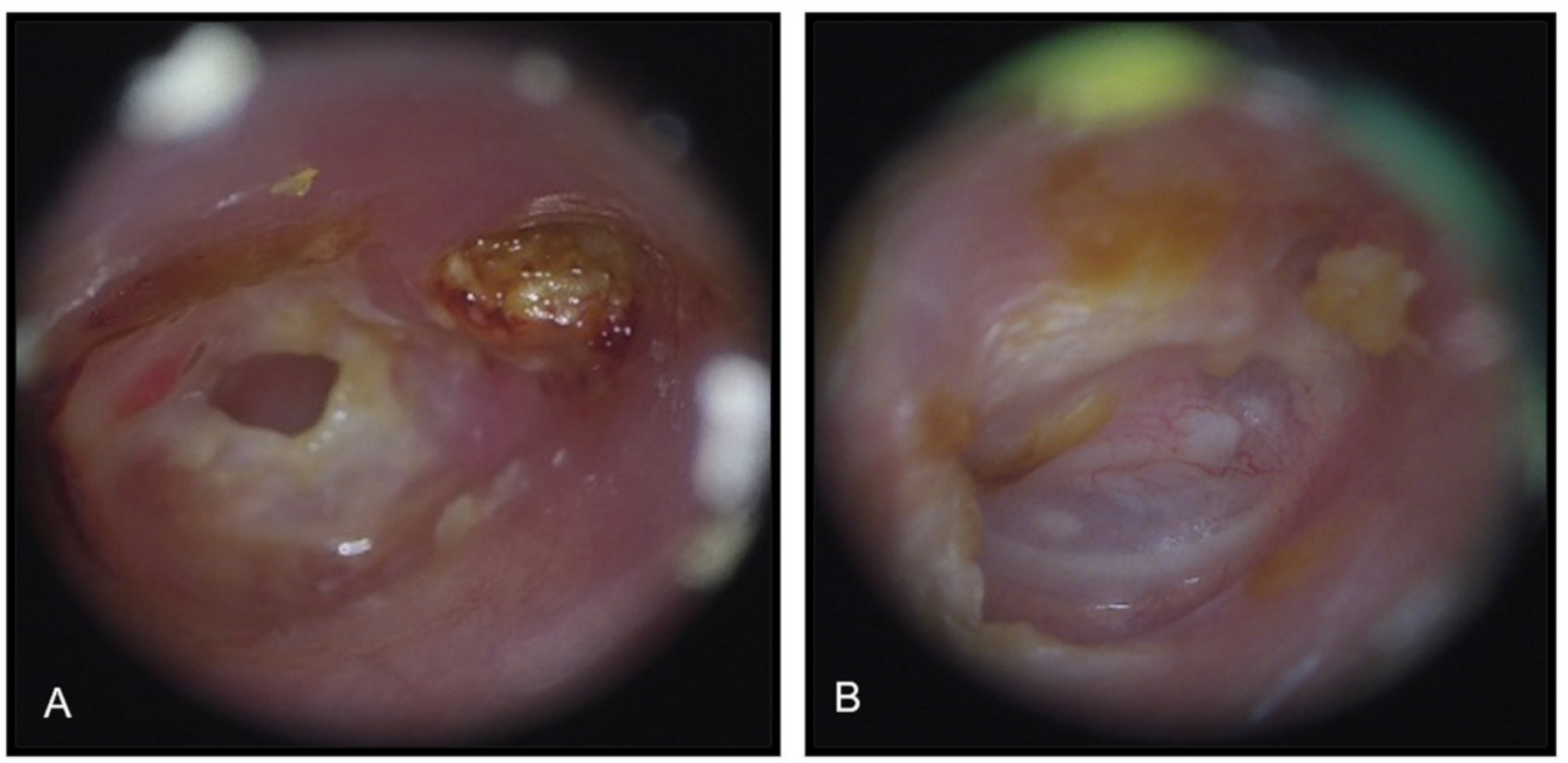


\section{Figure 4}

Preoperative and postoperative computed tomography

(A) Preoperative computed tomography showed scutum erosion and retraction pocket into the epitympanum. (B) Computed tomography taken 4 years after surgery found that the bone grafts use for EAC reconstruction is well maintained (arrow) with good aeration in the middle ear cavity.
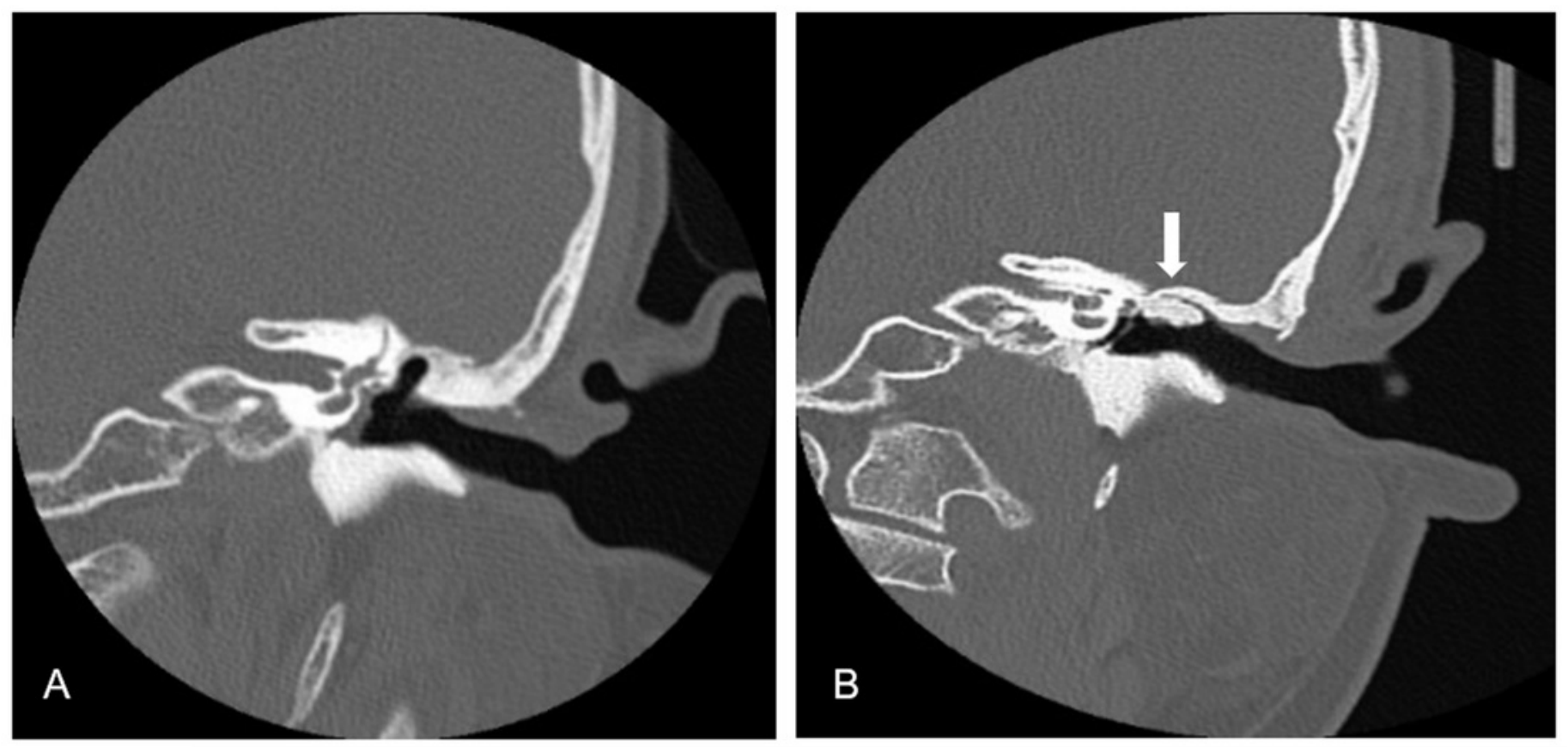